PAPER

\section{Tensorial description of quantum mechanics}

To cite this article: J Clemente-Gallardo and G Marmo 2013 Phys. Scr. 2013014012

View the article online for updates and enhancements.
Related content

Geometrical description of quantum mechanics-transformations and dynamics

G Marmo and G F Volkert

The variational method for density states a geometrical approach Armando Figueroa, Octavio Castaños,

Ramón López-Peña et al.

Tensorial dynamics on the space of quantum states

J F Cariñena, J Clemente-Gallardo, J A Jover-Galtier et al.

\section{Recent citations}

Tensorial dynamics on the space of quantum states

J F Cariñena et al

Implications of nonsymmetric metric theories for particle physics: New interpretation of the Pauli coupling Ginés R. Pérez Teruel 


\title{
Tensorial description of quantum mechanics
}

\author{
J Clemente-Gallardo $^{1}$ and G Marmo ${ }^{2}$ \\ ${ }^{1}$ BIFI-Departamento de Física Teórica and Unidad Asociada IQFR-BIFI, Universidad de Zaragoza, \\ Edificio I+D-Campus Río Ebro, Mariano Esquillor s/n, E-50018 Zaragoza, Spain \\ ${ }^{2}$ Dipartimento di Scienze Fisiche dell' Università di Napoli 'Federico II' and INFN-Sezione di Napoli, \\ Complesso Universitario di Monte Sant'Angelo, via Cintia, I-80126 Napoli, Italy \\ E-mail: jesus.clementegallardo@bifi.es and marmo@na.infn.it
}

Received 10 September 2012

Accepted for publication 18 September 2012

Published 28 March 2013

Online at stacks.iop.org/PhysScr/T153/014012

\begin{abstract}
Relevant algebraic structures for the description of quantum mechanics in the Heisenberg picture are replaced by tensor fields on the space of states. This replacement introduces a differential geometric point of view which allows for a covariant formulation of quantum mechanics under the full diffeomorphism group.
\end{abstract}

PACS numbers: 03.65.Ca, 03.65.Aa

\section{Introduction}

One important motivation for a geometrical formulation of quantum mechanics originates from the following observation. The unification of fundamental interactions requires that we unify, preliminarily, two distinct theories: classical general relativity and quantum mechanics. It is reasonable to expect that what will eventually emerge will entail a substantial revision of both theories, which, we may expect, will emerge as merely approximations to some underlying new theory that encompasses them.

Other motivations for our geometrical formulation are the following. The space of pure states of a quantum system (usually identified with rays of a Hilbert space $\mathcal{H}$ or with rank-one projectors defined on $\mathcal{H}$ ) is not a linear space, rather it is a Hilbert manifold (i.e. a differential manifold whose tangent spaces (at each point) are endowed with a Hilbert space structure). Additional instances of manifolds of states, instead of a representation as elements of linear spaces, are provided by coherent states and generalized coherent states (see [1, 2]). Further, the space of entangled states, in composite systems, does not carry a linear structure and therefore a manifold point of view may help in studying their properties.

The manifold point of view requires us to consider nonlinear transformations, say diffeomorphisms, which provide a point of view for quantum mechanics closer to that achieved in the transition from special to general relativity. See [6] for a detailed review of the topic.

\section{The description of quantum mechanics}

\subsection{The carrier space of states and probability distributions}

The introduction of the geometrical point of view seems also more close to what is being measured in the laboratory. This formulation deals with expectation values of observables on the space of physical states. From this point of view, let us analyze the pairing between physical observables and physical states. We recall that in the Schrödinger picture we associate a Hilbert space with any quantum system, physical observables being Hermitian operators acting on it. It is important to note that the elements of the Hilbert space do not correspond directly to states of our physical system; instead, they can be considered as convenient entities which can be used to define probability distributions associated with Hermitian operators.

For instance, let us consider a resolution of the identity, associated with some self-adjoint operator. It can be written as

- $\sum_{k}\left|e_{k}\right\rangle\left\langle e_{k}\right|=\mathbb{I}_{\mathcal{H}}$ for $\left\{\left|e_{k}\right\rangle\right\}$ a discrete orthonormal basis of the Hilbert space $\mathcal{H}$, say eigenstates of an operator $E$, or

- $\int|a\rangle \mathrm{d} a\langle a|=\mathbb{I}_{\mathcal{H}}$ for $\{|a\rangle\}$ an orthonormal basis of eigenstates of an operator $A$ which is supposed to have a continuous spectrum.

The corresponding probability distribution is defined by

$$
\mathcal{P}_{\psi}^{E}(j)=\frac{\left\langle\psi \mid e_{j}\right\rangle\left\langle e_{j} \mid \psi\right\rangle}{\langle\psi \mid \psi\rangle}
$$


for the case of the discrete basis and by

$$
\mathrm{d} \mathcal{P}_{\psi}^{A}(a)=\frac{\langle\psi \mid a\rangle \mathrm{d} a\langle a \mid \psi\rangle}{\langle\psi \mid \psi\rangle}
$$

for the continuous case; in both cases for any Hilbert space vector $|\psi\rangle \in \mathcal{H}$.

An important spin-off of the replacement of states with probability distributions is that it calls for an extension of the notion of state. Indeed, if we consider states to be probability distributions, a convex combination of them is a probability distribution too and is therefore acceptable as a state of our physical system. Hence, they may be used to define expectation values of the operators representing physical observables.

By using the identification of pure states with rank-one projectors on the Hilbert space $\mathcal{H}$, as

$$
\mathcal{P}_{|\psi\rangle}=\frac{|\psi\rangle\langle\psi|}{\langle\psi \mid \psi\rangle}
$$

it follows that a convex combination

$$
\rho=\sum_{k} \lambda_{k} \frac{\left|\psi_{k}\right\rangle\left\langle\psi_{k}\right|}{\left\langle\psi_{k} \mid \psi_{k}\right\rangle}, \quad \lambda_{k} \geqslant 0, \quad \sum_{j} \lambda_{j}=1
$$

is also an admissible state. We shall denote by $\mathcal{S}$ the set of all possible states.

Each point $\rho \in \mathcal{S}$ is associated with a convex combination of the corresponding probability distributions:

$$
\sum_{k} \lambda_{k} \frac{\left\langle\psi_{k} \mid e_{j}\right\rangle\left\langle e_{j} \mid \psi_{k}\right\rangle}{\left\langle\psi_{k} \mid \psi_{k}\right\rangle}=\sum_{k} \lambda_{k} \mathcal{P}_{\left|\psi_{k}\right\rangle}^{E}(j)=\mathcal{P}_{\rho}^{E}(j) .
$$

Thus our starting point will be to consider the space of states as carrier space for the description of our quantum system and the space of real-valued functions on it as candidates for representing physical observables. Note that in this picture, as we give up the linear structure of the carrier space, there is no room for linear transformations, linear operators or linear superposition of vectors (see [11, 12]).

\subsection{The algebraic structures of the space of physical observables}

In order to identify the geometrical (tensorial) structures with which we should endow our carrier space, let us consider the structures which emerge from it if we start from the usual Hilbert space point of view.

The space of observables (i.e. of self-adjoint operators acting on $\mathcal{H}$ ) corresponds (up to an imaginary factor) to $\mathfrak{u}(\mathcal{H})$, the real Lie algebra of the unitary group of $U(\mathcal{H})$. It may be identified with its dual $\mathfrak{u}^{*}(\mathcal{H})$ by the (regular) scalar product defined as

$$
\begin{aligned}
& \langle\cdot \mid \cdot\rangle: \mathfrak{u}(\mathcal{H}) \times \mathfrak{u}(\mathcal{H}) \rightarrow \mathbb{R}, \quad\langle A \mid B\rangle=\frac{1}{2} \operatorname{Tr} A B, \\
& \forall A, B \in \mathfrak{u}(\mathcal{H}) .
\end{aligned}
$$

The corresponding isomorphism

$$
\zeta: \mathfrak{u}(\mathcal{H}) \rightarrow \mathfrak{u}^{*}(\mathcal{H})
$$

allows us to export the geometric and algebraic structures existing in each space, into the other. We can therefore consider the Poisson structure on the dual $\mathfrak{u}^{*}(\mathcal{H})$ as a tensor field on the space of observables (and therefore we can consider Hamiltonian dynamics), or extend the Jordan structure defined on $\mathfrak{u}(\mathcal{H})$ into its dual. We shall see this in the next section.

On the other hand, for arbitrary linear operators on the vector space $\mathcal{H}$, we know that the associative product of two operators can always be decomposed as the sum of a symmetric and a skew-symmetric part:

$$
A B=\frac{1}{2}(A B+B A)+\frac{1}{2}(A B-B A) \quad \forall A, B \in \operatorname{End}(\mathcal{H}) .
$$

If we restrict the operation to the space of Hermitian operators, we note that the symmetric part is an inner operation, while the skew-symmetric part is not. This can be amended by introducing the imaginary unit as a prefactor defining thus

$$
A \circ B=A B+B A, \quad[A, B]=-\mathrm{i}(A B-B A) .
$$

Both structures are then inner in the space of Hermitian operators. The skew-symmetric one defines a Lie-algebra structure, which corresponds to the unitary Lie algebra $\mathfrak{u}(\mathcal{H})$. The symmetric operation defines the notion of Jordan algebra. This structure was considered and completely analyzed in finite dimensions in the famous paper by Jordan et al [9].

Jordan algebras are commutative but not associative. They satisfy a weaker form of associativity, namely

$$
(A \circ A) \circ(B \circ A)=((A \circ A) \circ B) \circ A \quad \forall A, B \in \mathfrak{u}(\mathcal{H}) .
$$

Moreover, a compatibility condition between the two operations (the Jordan and Lie algebra) holds true, namely

$$
[A,(B \circ C)]=[A, B] \circ C+B \circ[A, C] .
$$

In the abstract setting, this additional relation together with the two structures defines what is called a Lie-Jordan structure (see $[3,5,10])$.

The further requirement

$$
A \circ(B \circ C)-(A \circ B) \circ C=K[[A, C], B],
$$

where $K$ is a suitable real number, allows us to reconstruct the associative product on the abstract space with elements $(A, B, C, \ldots)$.

\section{Tensorial description of the space of observables}

Our goal is to encode the algebraic structures existing for the set of physical observables at the level of the expectation-value functions. First, let us associate with any operator $A: \mathcal{H} \rightarrow \mathcal{H}$, the expectation value function $e_{A}: \mathcal{H} \rightarrow$ $\mathbb{C}$ defined as

$$
e_{A}(\psi)=\frac{\langle\psi \mid A \psi\rangle}{\langle\psi \mid \psi\rangle} .
$$

Any linear operator $A: \mathcal{H} \rightarrow \mathcal{H}$ can be decomposed as the sum of its real and imaginary parts

$$
A=A_{\mathrm{R}}+\mathrm{i} A_{\mathrm{I}} .
$$


Both, $A_{\mathrm{R}}$ and $A_{\mathrm{I}}$, are Hermitian operators. The expectation value functions associated with Hermitian operators are real valued.

\subsection{Poisson, Jordan and associative structures}

We may introduce dynamics in this framework with the help of the Ehrenfest picture and write the equations of motion in terms of the expectation value functions:

$$
\frac{\mathrm{d}}{\mathrm{d} t} e_{A}(\psi)=\frac{1}{\mathrm{i} \hbar} \frac{\langle\psi \mid(H A-A H) \psi\rangle}{\langle\psi \mid \psi\rangle}=\frac{\langle\psi \mid[H, A] \psi\rangle}{\langle\psi \mid \psi\rangle},
$$

where

$$
[H, A]:=\frac{1}{\mathrm{i} \hbar}(H A-A H) .
$$

Equation (12) uses the Lie-algebraic structure defined by the commutator on the space of Hermitian operators.

Observables also give rise to observed quantities with associated indetermination, i.e. the variance

$$
\Delta A(\psi)=\frac{\left\langle\psi \mid A^{2} \psi\right\rangle}{\langle\psi \mid \psi\rangle}-\left(\frac{\langle\psi \mid A \psi\rangle}{\langle\psi \mid \psi\rangle}\right)^{2},
$$

and higher order moments. The Jordan structure of the space of observables is reflected in this type of objects.

Finally, we can also introduce the associative product of the linear operators on $\mathcal{H}$ into the expectation-value functions. By using equation (6), it is immediate to write

$$
e_{A} \star e_{B}(\psi):=e_{A B}(\psi)=\frac{1}{2} e_{A \circ B}(\psi)+\frac{\mathrm{i} \hbar}{2} e_{[A, B]}(\psi) .
$$

\subsection{Digression: algebraic structures described by tensor fields}

Given a vector space $V$ and a binary bilinear product

$$
\mathcal{B}: V \times V \rightarrow V
$$

we can replace $V$ by its bidual, i.e. the set of linear functions on the dual space

$$
V \hookrightarrow \mathcal{F}\left(V^{*}\right) ; \quad v \mapsto \hat{v} .
$$

Then, we can define a tensor field on $V^{*}$ which, at the point $\xi \in V^{*}$, takes the form

$$
\tau_{\mathcal{B}}\left(\mathrm{d} \hat{v}_{1}, \mathrm{~d} \hat{v}_{2}\right)(\xi)=\xi\left(\mathcal{B}\left(v_{1}, v_{2}\right)\right) \quad \forall v_{1}, v_{2} \in V, \xi \in V^{*},
$$

where $\hat{v}_{1}$ and $\hat{v}_{2}$ are the linear functions belonging to $\mathcal{F}\left(V^{*}\right)$ which are defined by $v_{1}$ and $v_{2}$, respectively.

If we want a description solely in terms of structures on the space of expectation value functions, and using our experience from classical mechanics, we should seek Poisson and Jordan structures defined on that space (we may call them quantum Poisson (quantum Jordan) structure $(s)$ ), such that

$$
\left\{e_{A}, e_{B}\right\}(\psi)=e_{[A, B]}(\psi) ; \quad\left\{e_{A}, e_{B}\right\}_{+}(\psi)=e_{A \circ B}(\psi) .
$$

As a result, the carrier space of states gets endowed with a contravariant tensor $\Lambda$ which encodes the Poisson structure and with a contravariant tensor $G$ which encodes the Jordan product.
In our case, the vector space $V$ represents the space of expectation-value functions, which are functions on the carrier space of the system. This carrier space may be identified with the convex body $\mathcal{S}$ defined by equation (3), and therefore may be considered to be contained in $\mathfrak{u}^{*}(\mathcal{H})$. Denoting by $\rho \in \mathcal{S}$ a generic state, we can write the expectation value function associated with a Hermitian operator $A$ as

$$
e_{A}(\rho)=\rho(A)=\operatorname{Tr}(\rho A),
$$

where we used the same symbol $\rho$ to represent the Hermitian operator which is associated with the state $\rho \in \mathfrak{u}^{*}(\mathcal{H})$ via the isomorphism $\zeta$ in equation (5). This is a linear function on the dual space $\mathfrak{u}^{*}(\mathcal{H})$, and thus it is associated (because of the isomorphism defined by equation (5)) with the Hermitian operator $A$. Thus, the tensor associated with a bilinear binary structure $\mathcal{B}: \mathfrak{u}(\mathcal{H}) \times \mathfrak{u}(\mathcal{H}) \rightarrow \mathfrak{u}(\mathcal{H})$, where $(A, B) \mapsto$ $\mathcal{B}(A, B)$, reads

$$
\tau_{\mathcal{B}}\left(d e_{A}, d e_{B}\right)(\rho)=\rho(\mathcal{B}(A, B)) .
$$
for:

Hence we can now define the two tensors we are looking

- the Poisson tensor associated with the Lie-algebra structure that is defined on $\mathfrak{u}^{*}(\mathcal{H})$ and reads

$$
\Lambda\left(d e_{A}, d e_{B}\right)(\rho)=\rho([A, B]) ;
$$

- the Jordan tensor associated with the symmetric operation ${ }^{\circ}$ defined on $\mathfrak{u}^{*}(\mathcal{H})$ :

$$
G\left(d e_{A}, d e_{B}\right)(\rho)=\rho(A \circ B) .
$$

Therefore, we have been able to reconstruct the algebraic structures of the space of physical observables as tensorial objects defined on the space of expectation-value functions. Further details can be found in [7, 8].

\subsection{Characterizing the observables}

The question we want to answer now is the following: if we are given a function $F$ in the space of states $\mathcal{S}$, how do we know that it is associated with an observable?

We can answer the question by using the tensors $\Lambda$ and $G$ we introduced in the previous section. Consider the Hamiltonian vector field $X_{F}$ which is associated with $F$ via the Poisson tensor $\Lambda$. This tensor field can be considered as the infinitesimal generator of a diffeomorphism acting on $\mathcal{S}$. Then, it can be proved (see [4]) that

Theorem 1. F represents a physical observable if and only if the associated Hamiltonian vector field $X_{F}$ preserves the tensor $G$, i.e.

$$
\mathcal{L}_{X_{F}} G=0 \text {. }
$$

In this way, we can affirm that the space of states $\mathcal{S}$ (with its structure as a stratified manifold, see; [7, 8]), along with the tensors $\Lambda$ and $G$, completely encodes quantum mechanics at a geometric level. The description allows us to consider generic transformations, and not just the linear ones, since the emerging description is covariant under the full diffeomorphism group. 


\section{Example: the two-level system}

Let us illustrate our arguments in the simplest but nontrivial example: the two-level system.

In this case, the set of Hermitian operators correspond (modulo a multiplication by the imaginary unit) to the Lie algebra $\mathfrak{u}(2)$. We can consider the basis defined by the four Pauli matrices $\left\{\sigma_{\mu}\right\}_{\mu=0,1,2,3}$ and represent the corresponding coordinates as

$$
Y_{\mu}(A)=\frac{1}{2} \operatorname{Tr}\left(A \sigma_{\mu}\right)
$$

The convex body of states $\mathcal{S}$ is defined by the set of points

$$
\rho=\frac{1}{2}\left(\sigma_{0}+\vec{x} \vec{\sigma}\right), \quad \vec{x} \vec{x} \leqslant 1 .
$$

Thus, physical states are in one-to-one correspondence with points of the Bloch ball, the points in the surface $(\vec{x} \vec{x}=1)$ representing the pure states.

As to the tensor fields, it is simple to write them down by using equation (19):

$$
\begin{aligned}
& G=\frac{\partial}{\partial Y_{0}} \otimes Y_{k} \frac{\partial}{\partial Y_{k}}+Y_{k} \frac{\partial}{\partial Y_{k}} \otimes \frac{\partial}{\partial Y_{0}} \\
& +Y_{0}\left(\frac{\partial}{\partial Y_{0}} \otimes \frac{\partial}{\partial Y_{0}}+\frac{\partial}{\partial Y_{k}} \otimes \frac{\partial}{\partial Y_{k}}\right), \\
& \Lambda=\epsilon^{j k l} Y_{j} \frac{\partial}{\partial Y_{k}} \otimes \frac{\partial}{\partial Y_{l}} .
\end{aligned}
$$

By using this last tensor, we can, for instance, recover at the level of $\mathfrak{u}^{*}(2)$, the angular-momentum commutation relations:

$$
\left\{Y_{k}, Y_{l}\right\}=\epsilon_{k l m} Y_{m}
$$

By using the composition $\mathcal{A}=G+\mathrm{i} \Lambda$, we can recover the associative product of operators at the level of the expectation value functions. Thus, we can write

$$
e_{A} \star e_{B}:=e_{A B}=\mathcal{A}_{\mu \nu}^{\xi} Y_{\xi} \frac{\partial e_{A}}{\partial Y_{\mu}} \frac{\partial e_{B}}{\partial Y_{\nu}} .
$$

At this point, we can show how it is possible to use nonlinear transformation on the carrier space. We can perform the transition to polar spherical coordinates $\left(y_{0}, r, \theta, \phi\right)$

$$
x=r \sin \theta \sin \phi, \quad y=r \sin \theta \cos \phi, \quad z=r \cos \theta,
$$

and write the expression for the tensors in these coordinates. For instance, the Poisson tensor becomes

$$
\Lambda=\frac{1}{r \sin \theta} \frac{\partial}{\partial \theta} \wedge \frac{\partial}{\partial \phi}
$$

while the Jordan one reads as

$$
\begin{aligned}
G= & r \frac{\partial}{\partial y_{0}} \otimes \frac{\partial}{\partial r}+r \frac{\partial}{\partial r} \otimes \frac{\partial}{\partial y_{0}} \\
& +y_{0}\left(\frac{\partial}{\partial y_{0}} \otimes \frac{\partial}{\partial y_{0}}+\frac{\partial}{\partial r} \otimes \frac{\partial}{\partial r}\right. \\
& \left.+\frac{1}{r^{2}} \frac{\partial}{\partial \theta} \otimes \frac{\partial}{\partial \theta}+\frac{1}{r^{2} \sin ^{2} \theta} \frac{\partial}{\partial \phi} \otimes \frac{\partial}{\partial \phi}\right) .
\end{aligned}
$$

These tensorial expressions show that the equations of motion, or the indetermination relations associated with pairs of expectation value functions, may be written in any coordinate system.

\section{Acknowledgments}

GM would like to acknowledge the support provided by the Santander/UCIIIM Chair of Excellence programme 2011-2012. J C-G has been partially supported by DGA Grant (24/1) and MICINN Grant (Fis2009-12648-C03-02).

\section{References}

[1] Aniello P, Clemente-Gallardo J, Marmo G and Volkert G F 2010 Classical tensors and quantum entanglement. I: pure states Int. J. Geom. Methods Mod. Phys. 7485

[2] Aniello P, Clemente-Gallardo J, Marmo G and Volkert G F 2011 Classical tensors and quantum entanglement. II: mixed states Int. J. Geom. Methods Mod. Phys. 8853

[3] Chruscinski D and Marmo G 2009 Remarks on the GNS construction and the geometry of quantum states Open Syst. Inform. Dyn. 16157

[4] Cirelli R, Mania A and Pizzocchero L 1994 A functional representation for noncommutative $C^{*}$-algebras $R e v$. Math. Phys. 6675

[5] Emch G G 1984 Mathematical and Conceptual Foundations of 20th Century Physics (Amsterdam: North-Holland)

[6] Ercolessi E, Marmo G and Morandi G 2010 From the equations of motion to the canonical commutation relations Riv. Nuovo Cimento 33(8-9) 401

[7] Grabowski J, Kus M and Marmo G 2005 Geometry of quantum systems: density states and entanglement J. Phys. A: Math. Gen. 3810217

[8] Grabowski J, Kus M and Marmo G 2006 Symmetries, group actions, and entanglement Open Syst. Inform. Dyn. 13343

[9] Jordan P, von Neumann J and Wigner E 1934 On an algebraic generalization of the quantum mechanical formalism Anal. Math. 3529

[10] Landsman N P 1998 Mathematical Topics between Classical and Quantum Mechanics (New York: Springer)

[11] Manko V I, Marmo G, Sudarshan E C G G and Zaccaria F 2000 Inner composition law of pure states as a purification of impure states Phys. Lett. A 27331

[12] Manko V I, Marmo G, Sudarshan E C G G and Zaccaria F 2002 Interference and entanglement: an intrinsic approach J. Phys. A: Math. Gen. 357137 